\title{
ON APPROXIMATION BY NONVANISHING FUNCTIONS
}

\author{
O. J. FARRELL
}

1. The measure of approximation. Let the function $f(z)$ be analytic and uniformly limited and have $k$ zeros interior to a simply connected region $G$ of the plane of the complex variable $z=x+i y$. The set of functions analytic interior to $G$ and which vanish either identically or not at all interior to $G$ form a closed set $S[1$, p. 343]. For every function $F(z)$ uniformly limited and of class $S$ in $G$ we set

$$
M(F)=\text { l.u.b. }[|F(z)-f(z)|, z \text { in } G]
$$

and let $M$ denote the greatest lower bound of all $M(F)$. Walsh has shown [1, pp. 344-346] that there exists a function in $S$, call it $F^{*}(z)$, for which $M\left(F^{*}\right)=M$. In certain situations he has found the precise value of $M$ as well as functions $F^{*}(z)$ of best approximation. In some instances he has exhibited an infinity of functions $F^{*}(z)$ of best approximation.

The purpose of the present paper is (1) to put an appraisal on $M$, (2) to present two theorems on the number of zeros in $G$ of functions which approximate closer to $f(z)$ than the lower appraisal on $M$.

2. Appraisal of $M$. Consider a region $G$, a function $f(z)$, the set $S$ of functions $F(z)$ and the measure $M$ of best approximation to $f(z)$ by functions of class $S$, all as described above in $\S 1$. Let the $k$ zeros of $f(z)$ in $G$ occur as follows: $k_{1}$ zeros at $z=a_{1}, k_{2}$ zeros at $z=a_{2}, \cdots, k_{m}$ zeros at $z=a_{m}$, where $k_{1}+k_{2}+\cdots+k_{m}=k$. Let $R$ denote the Riemann configuration over the w-plane $[2$, p. 130] onto which $G$ is mapped by $w=f(z)$. Let the points $w_{1}, w_{2}, \cdots, w_{m}$ on $R$, all with affix $w=0$, represent respectively $f\left(a_{1}\right), f\left(a_{2}\right), \cdots, f\left(a_{m}\right)$. At each point $w_{i}$ of the set $w_{1}, w_{2}, \cdots, w_{m}$ consider the radius $D_{k_{i}}\left(w_{i}\right)$ of $k_{i}$-valence there [2, pp. 161-162]. Let $D_{0}$ denote the greatest number to be found among the $D_{k_{i}}\left(w_{i}\right)$.

Any point $w$ on the $w$-plane such that there is a point $P$ of $R$ whose affix is $w$ will be said (as in [2]) to be covered by $P$. Let $r_{0}$ denote the radius of the largest circle $K_{0}$ of all circles $K$ centered at $w=0$ such that every point within $K$ is covered by at least point of $R$. On $K_{0}$ itself there will be at least one point which is not covered by any point of $R$. It is seen that $D_{0} \leqq r_{0}$. Then we have the following appraisal of $M$.

THEOREM 1. $D_{0} \leqq M \leqq r_{0}$.

Received by the editors February 10, 1959 and, in revised form, March 19, 1959. 
Proof. Let $w=b$ be a point on $K_{0}$ which is not covered by any point of $R$. It follows at once that the function $F(z)=f(z)-b$ is in class $S$ and such that $M(F)=|b|=r_{0}$. It remains to prove that $D_{0} \leqq M$. Let $g(z)$ be any function which is analytic interior to $G$ and such that

$$
|g(z)-f(z)| \leqq H<D_{0}, \quad z \text { in } G .
$$

Cut through $R$ with a circular biscuit cutter of radius $C$, where $H<C<D_{0}$, so that the centers of circular sheets thus cut from $R$ have affix $w=0$. Let $z=q(w)$ denote the inverse of $w=f(z)$ and consider the transform under $z=q(w)$ of the $k_{h}$-sheeted circle [2, p. 159] of radius $C$ inside the biscuit cutter with sheets hanging together at $w_{h}$, where $w_{h}$ is a point of the set $w_{1}, w_{2}, \cdots, w_{m}$ such that $D_{k_{h}}\left(w_{h}\right)$ $=D_{0}$. This transform is a simply connected region $Q_{h}$ lying in $G$ and bounded by a contour $J_{h}$ also lying in $G$ [2, p. 164]. On $J_{h}$ we have $|f(z)|=C$ and $|g(z)-f(z)|<C$. It follows by Rouche's Theorem $\left[1\right.$, p. 6] that the function $g(z)$ has precisely $k_{h}$ zeros within $J_{h}$. Thus every function $g(z)$ for which l.u.b. $[|g(z)-f(z)|, z$ in $G]<D_{0}$ vanishes in $G$. Consequently $M$ can not be less than $D_{0}$.

3. Number of zeros of approximating functions. By following through with the method employed in the proof of Theorem 1 we obtain a result on the number of zeros of approximating functions as follows. Let the distinct numbers to be found among the $D_{k_{i}}\left(w_{i}\right)$ arranged in order be $D_{0}>D_{1}>D_{2}>\cdots>D_{p}$. Then we have the following theorem.

Theorem 2. Let $f(z)$ be analytic and uniformly limited interior to a simply connected region $G$ and have $k$ zeros in $G$ distributed as described above in $\$ 2$. Let $g(z)$ be analytic interior to $G$ and such that

$$
|g(z)-f(z)| \leqq C<D^{*}, \quad z \text { in } G,
$$

where $D^{*}$ is one of the numbers $D_{0}, D_{1}, D_{2}, \cdots, D_{p}$. Then $g(z)$ has at least as many zeros in $G$ as the sum of the $k_{i}$ for which $D_{k_{i}}\left(W_{i}\right) \geqq D^{*}$.

The proof of Theorem 2 is omitted, since it is essentially contained (except for the addition of the pertinent $k_{i}$ which provide the count of the zeros of $g(z)$ within the contours $J_{i}$ ) in the latter part of the proof of Theorem 1 .

We observe in particular that if

$$
\text { l.u.b. }[|g(z)-f(z)|, z \text { in } G]<D_{p},
$$

it follows at once by Theorem 2 that $g(z)$ has at least as many zeros 
in $G$ as has $f(z)$. Indeed, we can make this observation precise, as shown in our next theorem.

TheOREM 3. If $g(z)$ is analytic interior to $G$ and such that

$$
|g(z)-f(z)| \leqq L<D_{p}, \quad z \text { in } G,
$$

then $g(z)$ has precisely as many zeros in $G$ as has $f(z)$.

Proof. As we have already indicated, it follows by the method of proof used in Theorem 1 that $g(z)$ has precisely $k$ zeros situated interior to the regions (bounded by the contours $J_{1}, J_{2}, \cdots, J_{m}$ ) which are the transforms by $z=q(w)$ of the $m$ simply connected multisheeted circles (including single-sheeted circles, if any, corresponding to simple zeros of $f(z)$, if any) cut from $R$ by a circular biscuit cutter of radius $r$, where $L<r<D_{p}$ and the centers of the circles all have affix $w=0$. If $g(z)$ were to have more than $k$ zeros in $G$, all zeros other than the $k$ zeros just mentioned would have to lie not interior to the contours $J_{1}, J_{2}, \cdots, J_{m}$. Suppose there were such an additional zero at $z=a$. Then the point $w=f(a)$ on $R$ would not lie interior to the biscuit cutter; and we would have $|f(a)| \geqq r>L$. But $g(a)=0$. This would make $|g(a)-f(a)|>L$, contrary to hypothesis. Therefore $g(z)$ has precisely $k$ zeros in $G$.

\section{REFERENCES}

1. J. L. Walsh, Interpolation and approximation by rational functions in the complex domain, Amer. Math. Soc. Colloquium Publications, 2d ed. vol. 20, 1956.

2. W. Seidel and J. L. Walsh, On the derivatives of functions analytic in the unit circle and their radii of univalence and p-valence, Trans. Amer. Math. Soc. vol. 52 (1942) pp. 128-216.

UNion COLlege 